\title{
The prognostic significance of neutrophil-to-lymphocyte ratio at different time points in patients with hepatocellular carcinoma receiving liver resection
}

\author{
Tianxing Dai ${ }^{1}$, Guozhen Lin ${ }^{1}$, Mingbin Deng ${ }^{1}$, Shuguang Zhu ${ }^{1,2}$, Haibo Li ${ }^{1,2}$, Jia Yao ${ }^{1,2}, \mathrm{Hua} \mathrm{Li}^{1,2}$, \\ Wei Liu ${ }^{3}$, Yang Yang ${ }^{1,2}$, Guihua Chen ${ }^{2,3}$, Guoying Wang ${ }^{1,2}$ \\ ${ }^{1}$ Department of Hepatic Surgery and Liver Transplant Program, The Third Affiliated Hospital of Sun Yat-Sen University, Guangzhou 510630, \\ China; ${ }^{2}$ Organ Transplantation Institute of Sun Yat-Sen University, Guangzhou 510630, China; ${ }^{3}$ Guangdong Key Laboratory of Liver Disease \\ Research, The Third Affiliated Hospital of Sun Yat-Sen University, Guangzhou 510630, China \\ Contributions: (I) Conception and design: T Dai, G Wang; (II) Administrative support: G Wang; (III) Provision of study materials or patients: Y \\ Yang, G Chen, G Wang; (IV) Collection and assembly of data: T Dai, G Lin, M Deng; (V) Data analysis and interpretation: S Zhu, H Li, J Yao; (VI) \\ Manuscript writing: All authors; (VII) Final approval of manuscript: All authors. \\ Correspondence to: Guoying Wang. Department of Hepatic Surgery and Liver Transplant Program, The Third Affiliated Hospital of Sun Yat-Sen \\ University, 600 Tianhe Road, Guangzhou 510630, China. Email: wanggy3@126.com.
}

Background: Neutrophil-to-lymphocyte ratio (NLR) has been demonstrated a significant association with the prognosis of hepatocellular carcinoma (HCC). The current study aimed to evaluate the prognostic value of NLR at different time points in HCC patients receiving liver resection.

Methods: Data were retrospectively collected from 195 HCC patients receiving liver resection. The preoperative NLR (pre-NLR), postoperative NLR (post-NLR) and corresponding changes of NLR (NLRc) at different time points were calculated. The disease-free survival (DFS) and overall survival (OS) was calculated by the Kaplan-Meier method and compared by the log-rank test. Both univariate and multivariate analyses were performed to evaluate their prognostic values for DFS and OS. And the prognostic significance of pre-NLR, post-NLRs, and NLRcs were further evaluated with subgroup analysis and with early and late recurrence of $\mathrm{HCC}$.

Results: Pre-NLR was not significantly correlated with DFS or OS (both $\mathrm{P}>0.05$ ), whereas higher postNLR at 4-8 weeks [NLR (4-8 w)] and 3-6 months [(NLR $(3-6 \mathrm{~m})]$ predicted worse DFS $(\mathrm{P}=0.023$ and $\mathrm{P}<0.001$, respectively) and $\mathrm{OS}(\mathrm{P}=0.012$ and $\mathrm{P}=0.001$, respectively). The value of area under the curve (AUC) of NLR (3-6 m) were higher than NLR (4-8 w) for DFS (0.656 vs. 0.572) and OS (0.650 vs. 0.621). Multivariate analyses showed that NLRc (4-8 w) was not a significant predictor of DFS $(\mathrm{P}=0.369)$ or OS ( $\mathrm{P}=0.173)$, while the NLRc (3-6 m) with 25\% increase was found to be an independent factor for adverse DFS in patients with HCC $(\mathrm{P}=0.041)$. The AUC of NLRc $(3-6 \mathrm{~m})$ for DFS was 0.600. Subgroup analysis showed NLR $(3-6 \mathrm{~m})$ was significantly corrected to DFS $(\mathrm{P}<0.001)$ and $\mathrm{OS}(\mathrm{P}=0.001)$ in patients with cirrhosis. And NLR (3-6 m) also showed with significant correlation with early recurrence $(\mathrm{P}<0.001)$, while NLR (4-8 w) was found with significant association both with early and late recurrence $(\mathrm{P}=0.037$ and $\mathrm{P}=0.027$, respectively).

Conclusions: The post-NLRs are significant predictors of clinical outcome in HCC patients receiving liver resection, and post-NLR and NLRc with a relatively long-term interval after operation have better prognostic values.

Keywords: Disease-free survival (DFS); hepatocellular carcinoma (HCC); neutrophil-to-lymphocyte ratio (NLR); overall survival (OS); prognosis

Submitted Jun 26, 2019. Accepted for publication Nov 05, 2019.

doi: $10.21037 /$ tcr.2019.11.29

View this article at: http://dx.doi.org/10.21037/tcr.2019.11.29 


\section{Introduction}

Hepatocellular carcinoma (HCC), the most common type of malignant liver tumors, ranks sixth in incidence and third in mortality among all cancers, which causes a serious medical burden worldwide $(1,2)$. The risk factors of HCC are diverse with different etiologies, while the prevalence of chronic liver disease accounts for more than $80 \%$, especially with viral hepatitis-related cirrhosis (hepatitis B and/or C) (3). Despite the progression either in diagnostic modalities or surveillance programs of HCC, the proportion of patients available to curative treatments with early stage is still less than $30 \%(3,4)$. And according to the Barcelona clinic liver cancer (BCLC) system, liver resection, transplantation, and locoregional ablation are the potential curative therapies widely accepted (4). But the prognosis of these patients remains unsatisfactory with 5 -year survival rates of $50-70 \%$, and the high recurrence rate of HCC is also an important problem that cannot be ignored (3). Therefore, it's essential to find novel biomarkers to recognize patients with high risk of tumor recurrence and then take preventive measures to prolong the recurrence-free survival and overall survival.

Recently, the relationship between systematic inflammation and tumor biology has been demonstrated by an increased evidence in many cancers $(5,6)$. And studies have shown that systemic inflammatory responses can promote angiogenesis, DNA damage, and tumor invasion through the upregulation of cytokines. The neutrophil-tolymphocyte ratio (NLR), which was considered as a credible indicator of systemic inflammatory response, has shown an association with prognosis in numerous malignancies. In addition, NLR has also been investigated for its prognostic role in HCC. Most of these studies showed that an elevated pretreatment NLR predicted a poor disease-free and overall survival of HCC after different treatments (7-13), while other studies failed to demonstrate such an association (14-16). Therefore, the prognostic role of NLR in HCC needs further elucidation.

NLR usually changes along with the course of disease, especially after treatment, which may reflect the status shift of inflammatory response. Recently, several studies have focused on the significance of dynamics of NLR after treatment in several solid malignancies, such as renal cancer $(17,18)$, non-small cell lung cancer (19), and gastric cancer (20), which showed a better prognostic role of the change in NLR than pretreatment NLR and could act as a biomarker for efficacy. But the data of postoperative NLR differs from the timing after surgery in different studies and the optimal duration of postoperative NLR remains unclear. Moreover, the roles of postoperative NLR and change of NLR in HCC were also evaluated in several studies (15,16,21-23). But there exist some limitations: (I) most studies chose the NLR at one month after surgery as the postoperative NLR and carried the subsequent analysis; (II) the HCC patients were restricted to small HCCs or early stages HCCs in most studies; (III) there were few comparisons among preoperative NLR, postoperative NLR, and change of NLR in the prognostic value of HCC.

At present study, we performed a prognostic analysis in HCC patients who received liver resection as the first treatment, and subsequently evaluate the prognostic value of NLR at different time and the change of NLR after operation to find the optimal predictive parameter for HCC patients.

\section{Methods}

\section{Patients}

Patients, who were first diagnosed with HCC and treated in our department during March 2005 to May 2013, were collected from the inpatient database retrospectively. And the inclusion criteria were set as below: (I) adults with ages no less than eighteen years old; (II) received hepatectomy as the first treatment; (III) no pre-adjuvant therapies for HCC (TACE/ablation/sorafenib); (IV) pathologically proven HCC; (V) without extrahepatic or distant metastases; (VI) without other concurrent malignant tumors or hematological diseases; (VII) true, effective and complete inpatient data; (VIII) adequate follow-up. After reviewing the medical records, a total of 195 HCC patients met the criteria and were enrolled in our study.

\section{Clinicopathological data}

Personal basic information was collected with gender, age. Tumor-related data were reviewed by contrast-enhanced computed tomographic (CT) scans and/or magnetic resonance imaging (MRI) series and reports, including tumor size, nodule number and existence of vascular invasion. Besides, the presence of cirrhosis was evaluated both with radiological images and the histopathological reports. And the BCLC staging system was adopted to determine personalized staging for each patient.

Peripheral blood samples were obtained and examined within 1 week before surgery, 4-8 weeks and 3-6 months after surgery. The absolute neutrophil and lymphocyte counts were collected at each period. And the NLR was 
calculated by dividing the absolute neutrophil count by the absolute lymphocyte count. The pre-NLR, NLR (4-8 w) and NLR (3-6 m) represent NLR at different period mentioned above respectively. The NLR changes were calculated as \% changes by the following calculation: NLRc $=[($ NLR after surgery/NLR before surgery $)$ $-1)^{*} 100 \%$. Subsequently, we divided the NLRc into three groups with the change ratio $[\geq 25 \%$ decrease, no change ( $<25 \%$ decrease to $<25 \%$ increase), $\geq 25 \%$ increase]. The NLRc (4-8 w) and NLRc (3-6 m) represent the NLRc at 4-8 weeks and 3-6 months after surgery respectively.

\section{Follow-up}

Patients were followed up at the outpatient office with time sequences. The serum AFP levels, abdominal ultrasound, dynamic enhanced CT or MRI were performed according to the recommendations. And the follow-up programme began at the date of operation and ended with death or the time of last follow-up encompassed by this study (May 2013).

\section{Statistical analysis}

Continuous variables are reported as medians and interquartile ranges, while categorical variables are presented as frequencies and percentages. For NLR, the optimal cut-off value was determined by using receiver operating characteristic (ROC) curves. The area under the curve (AUC) was applied to evaluate the prediction value with respective sensitivity and specificity. Survival curves were constructed using the Kaplan-Meier method, and logrank tests were used for the univariate analysis. Variables that showed significant associations in the univariate analysis $(\mathrm{P}<0.05)$ were enrolled in a multivariate Cox regression model (forward stepwise method) to adjust the relationship between NLR (or NLRc) and DFS (or OS). All data analyses were carried out using the SPSS version 20.0 (IBM Crop., Chicago, IL, USA). All statistical tests were two-sided, and $\mathrm{P}<0.05$ was defined as statistically significant.

\section{Results}

\section{Patient characteristics}

A total of 195 HCC patients with complete medical records and follow-up data were involved in this study, including 174 males and 21 females. The baseline characteristics of these patients are shown in Table 1. The median age was 51 years (range: $42-59$ years). One hundred and twentysix $(64.6 \%)$ patients presented with cirrhosis, which were mainly related to hepatitis B and C. The median tumor size was $4.4 \mathrm{~cm}$ (range: $3.0-7.5 \mathrm{~cm}$ ). One hundred and fortyseven patients $(75.4 \%)$ presented with single tumor, while $48(24.6 \%)$ patients have multiple nodules. And 80 (41.0\%) patients were found with vascular invasion in contrastenhanced radiology examinations. According to the BCLC staging system, the numbers of patients with stage $0 \sim \mathrm{A}$, B, and C were 98 (50.3\%), 77 (39.5\%), and 20 (10.3\%), respectively. All the patients received R0 resection for their tumor in liver with a surgical margin $\geq 1 \mathrm{~cm}$ and confirmed with pathological examination. And well differentiation of the resected tumor was shown in $26(13.3 \%)$ patients, and $169(86.7 \%)$ patients presented with poor-moderate differentiation. During operation, 96 (49.2\%) patients had blood transfusion. Alpha-fetoprotein (AFP) was found increased ( $>400 \mathrm{ng} / \mathrm{mL}$ ) only in $65(33.3 \%)$ patients, while $130(66.7 \%)$ patients were with low level $(\leq 400 \mathrm{ng} / \mathrm{mL})$. The median levels of preoperative fibrinogen, platelet (PLT), alanine aminotransferase (ALT), albumin (ALB) and glutamyl transpeptidase (GGT) were $3.01 \mathrm{~g} / \mathrm{L}$ (range: $2.43-3.65 \mathrm{~g} / \mathrm{L}$ ), $175 \times 10^{9} / \mathrm{L}$ (range: $124 \times 10^{9}-231 \times 10^{9} / \mathrm{L}$ ), $36 \mathrm{U} / \mathrm{L}$ (range: 26-53 U/L), $39.6 \mathrm{~g} / \mathrm{L}$ (range: $37.5-42.5 \mathrm{~g} / \mathrm{L}$ ), and $63.0 \mathrm{U} / \mathrm{L}$ (range: $35.0-98.0 \mathrm{U} / \mathrm{L}$ ), respectively. The median levels of pre-NLR, NLR (4-8 w), and NLR (3-6 m) were 1.95 (range: 1.12-2.12), 1.47 (range: $1.12-2.12$ ), and 1.62 (range: $1.14-2.44$ ).

The median follow-up time was 25.5 months (range: 13.1-38.5 months). During the follow-up period, 121 $(62.1 \%)$ patients experienced recurrence, whereas 30 (15.4\%) patients died. The 1-, 3-, 5-year cumulative DFS rates was $58.0 \%, 34.4 \%$, and $27.1 \%$ respectively, whereas the 1-, 3-, and 5-year OS rates was $93.5 \%, 83.4 \%$, and $71.7 \%$, respectively (Figure 1).

\section{Evaluation of the NLRs and NLRcs for prognostic prediction}

We analyzed survival using receiver operating characteristics (ROC) curves to evaluate the probability of pre-NLR, NLR (4-8 w), NLR (3-6 m), NLRc (4-8 w) and NLRc (3-6 m) (shown in Figure 2).

Using DFS as the end point, the area under the ROC curve (AUC) for pre-NLR was 0.552 (95\% CI: $0.468-0.635, \mathrm{P}=0.226$ ), while the AUCs for postoperative NLR (4-8 w) and NLR (3-6 m) were 0.572 (95\% CI: 
Table 1 The baseline characteristics of 195 hepatocellular carcinoma patients

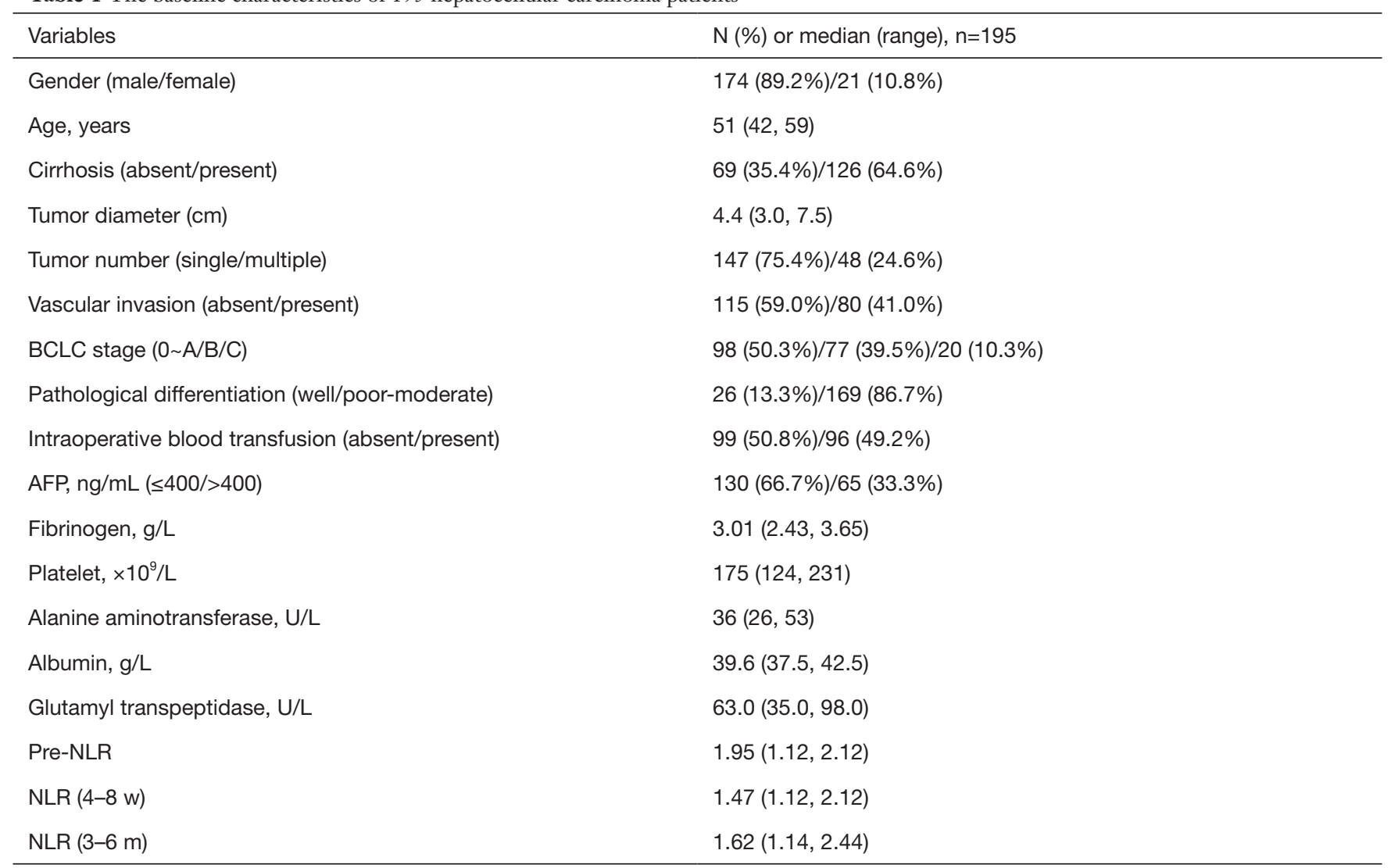

BCLC, Barcelona clinic liver cancer; AFP, $\alpha$-fetoprotein; NLR, neutrophil-to-lymphocyte ratio; Pre-NLR, preoperative NLR.

A

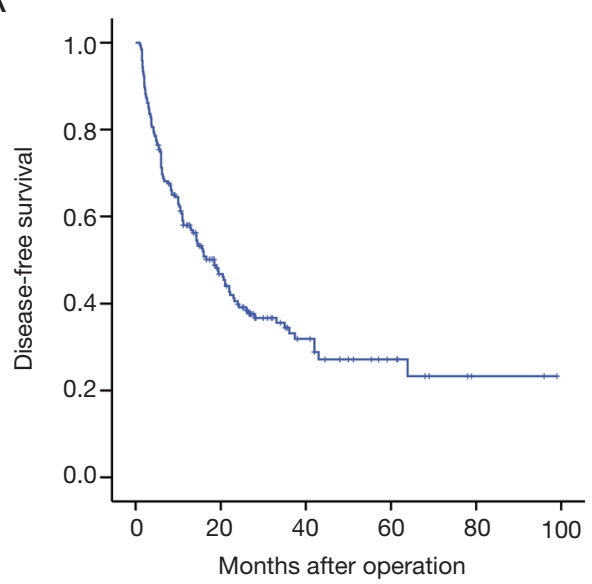

B

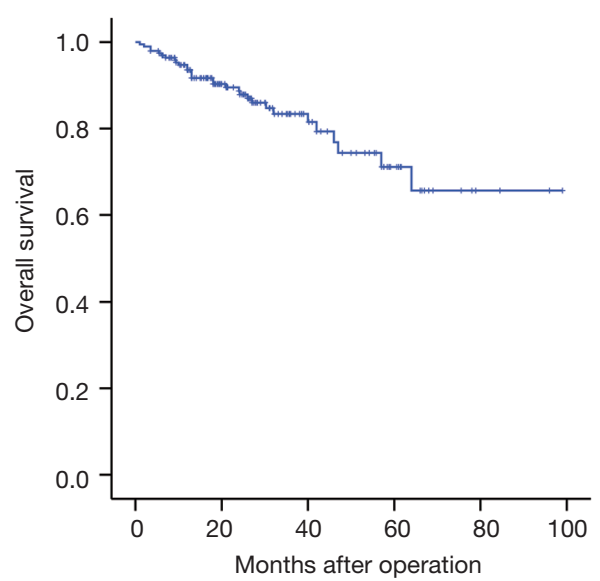

Figure 1 Kaplan-Meier curves for disease-free survival and overall survival of 195 patients with HCC receiving liver resection. Diseases-free survival (A), and overall survival (B). 
A

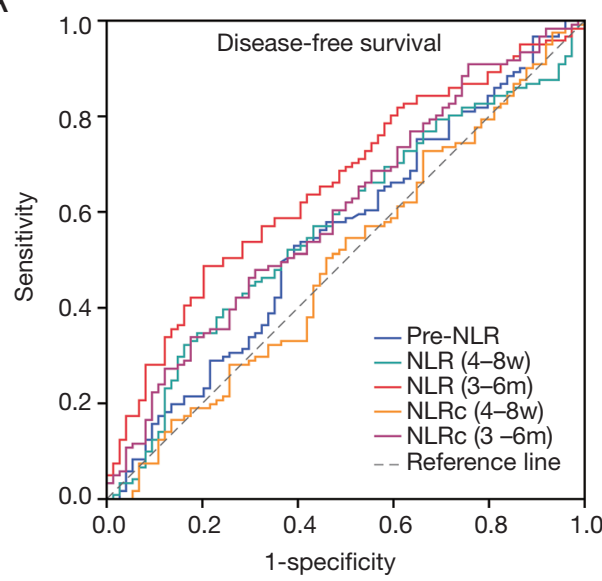

B

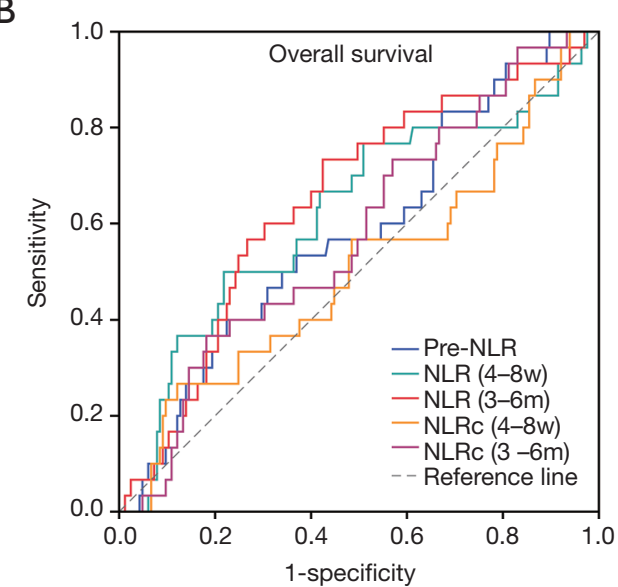

Figure 2 Receiver operating characteristic (ROC) curves for disease-free survival and overall survival of NLRs and NLRcs in HCC patients. Diseases-free survival (A), and overall survival (B).

$0.490-0.654, \mathrm{P}=0.093)$ and 0.656 (95\% CI: 0.578-0.733, $\mathrm{P}<0.001)$ respectively, which were both better than preNLR. Besides, the changes between postoperative and preoperative NLR, NLRc (4-8 w) and NLRc (3-6 m), showed different AUC with 0.498 (95\% CI: 0.413-0.582, $\mathrm{P}=0.954$ ) and 0.600 (95\% CI: $0.519-0.682, \mathrm{P}=0.042$ ), respectively. So, NLRc (3-6 $\mathrm{m}$ ) also presented with a higher AUC than pre-NLR.

As for OS, the AUC for pre-NLR was 0.582 (95\% CI: $0.473-0.692, P=0.152)$, whereas the AUCs for NLR (4-8 w) and NLR (3-6 m) were 0.621 (95\% CI: $0.504-0.739, \mathrm{P}=0.035)$ and 0.650 (95\% CI: $0.545-0.755$, $\mathrm{P}=0.009)$ respectively, which were both higher than Pre-NLR. And both the NLRc (4-8 w) and NLRc (3-6 m) showed a lower AUC with 0.505 (95\% CI: 0.386-0.625, $\mathrm{P}=0.924)$ and 0.579 (95\% CI: 0.4729-0.686, $\mathrm{P}=0.055)$ than pre-NLR.

\section{Prognostic values of NLRs and NLRcs for diseases-free and overall survival in HCC patients}

The optimal cut-off values of NLRs was determined by using ROC curves with Youden index and adjusted to 2 as an integral number for convenient clinical application. So, the pre-NLR, NLR (4-8 w) and NLR (3-6 m) were all divided into two groups with $\leq 2$ and $>2$. And the NLRc (4-8 w) and NLRc (3-6 m) were both divided into three groups as described above.

In univariate analyses, there was no significant relationship between pre-NLR and DFS (HR: 1.385, 95\%
CI 0.969-1.979, $\mathrm{P}=0.074$; Figure $3 A$ ), while significant relationships were found with the NLR (4-8 w) (HR: 1.742, 95\% CI: 1.193-2.544, P=0.004; Figure 3B) and NLR (3-6 m) (HR: 2.144, 95\% CI: 1.494-3.077, $\mathrm{P}<0.001$; Figure 3C). As for NLRcs, data showed that NLRc (4-8 w) was not significantly correlated with DFS $(\mathrm{P}=0.693$; Figure $3 D)$, while a significant relationship was found with the NLRc (3-6 m) $(\mathrm{P}=0.037$; Figure $3 E)$. Besides, no significant relationship between preNLR and OS (HR: 1.677, 95\% CI: 0.813-3.460, $\mathrm{P}=0.162$; Figure 4A) was found. However, the NLR (4-8 w) (HR: 2.838, 95\% CI: 1.379-5.841, $\mathrm{P}=0.005$; Figure 4B) and NLR (3-6 m) (HR: 3.122, 95\% CI: 1.500-6.497, P=0.002; Figure 4C) were significantly related to OS. As for NLRcs, neither the NLRc (4-8 w) $(\mathrm{P}=0.118$; Figure $4 D)$ nor the NLRc $(3-6 \mathrm{~m})(\mathrm{P}=0.241$; Figure $4 E$ ) was found significant relationship with OS. And the DFS of $\geq 25 \%$ increase in NLRc $(3-6 \mathrm{~m}$ ) was significantly lower than no change (HR: 1.640, 95\% CI: 1.037-2.594; $\mathrm{P}=0.034)$, whereas the DFS between $\geq 25 \%$ decrease and no change had no statistical significance (HR: 0.902, 95\% CI: $0.598-1.361 ; \mathrm{P}=0.623)$. Besides, tumor diameter $(>5 / \leq 5 \mathrm{~cm}$; HR: 2.096, 95\% CI: 1.457-3.016; $\mathrm{P}<0.001)$, tumor number (multiple/single; HR: 2.051, 95\% CI: 1.397-3.012; $\mathrm{P}<0.001$ ), vascular invasion (present/absent; HR: 1.972, 95\% CI: 1.374-2.830; $\mathrm{P}<0.001)$, BCLC stage (B C/0 A; HR: 2.141, 95\% CI: 1.481-3.094, $\mathrm{P}<0.001)$, pathological differentiation (present/absent; HR: 1.941, 95\% CI: 1.060-3.554, $\mathrm{P}=0.032$ ) and intraoperative blood transfusion (present/absent; HR: 1.468, 95\% CI: $1.024-2.105, \mathrm{P}=0.037)$ were found to be related to DFS significantly, while tumor diameter $(>5 / \leq 5 \mathrm{~cm}$; HR: $2.558,95 \%$ CI: $1.229-5.325, \mathrm{P}=0.012)$, vascular invasion 
A
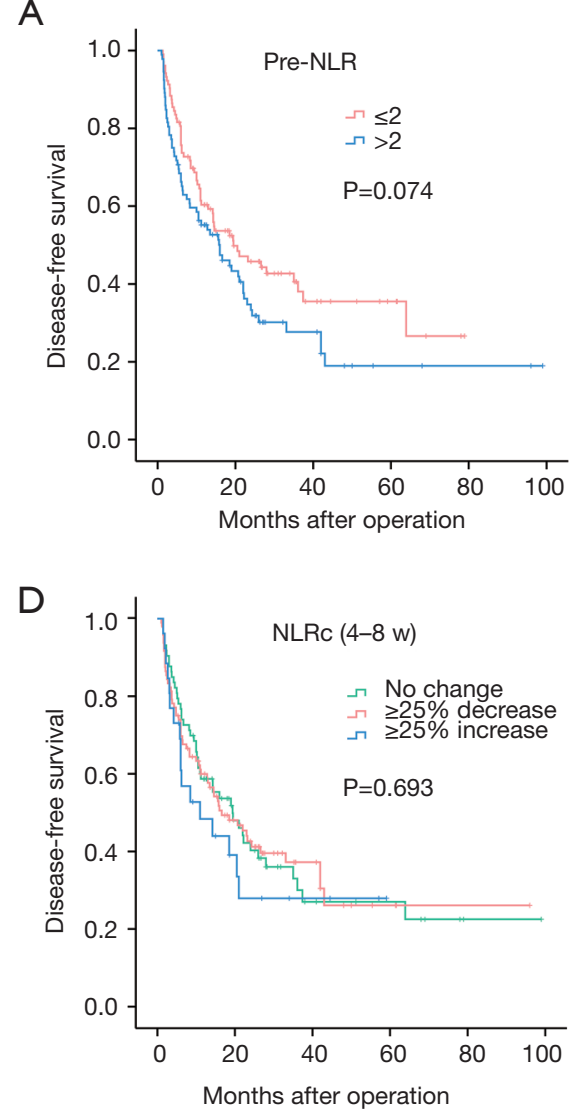

B

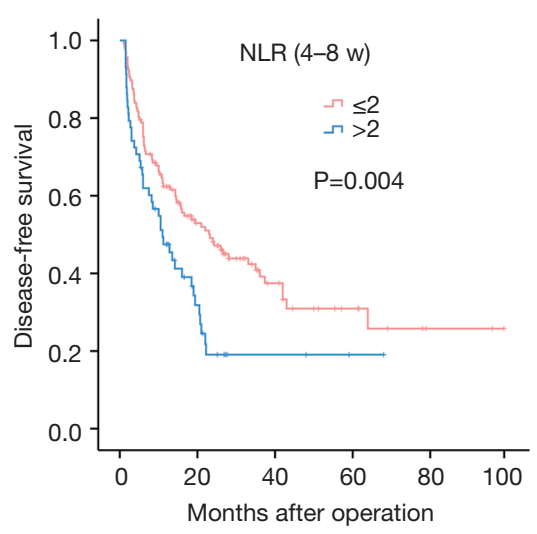

E

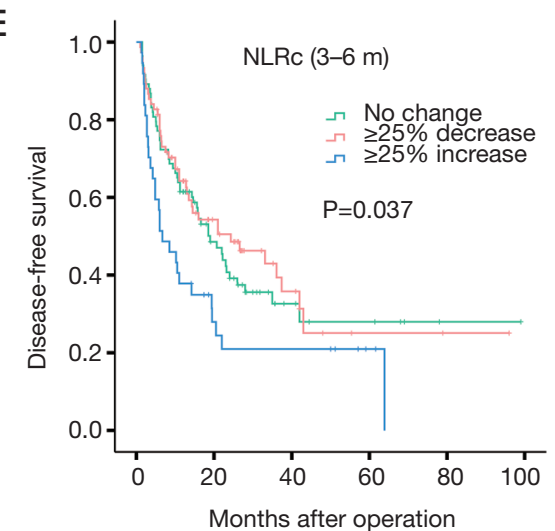

C

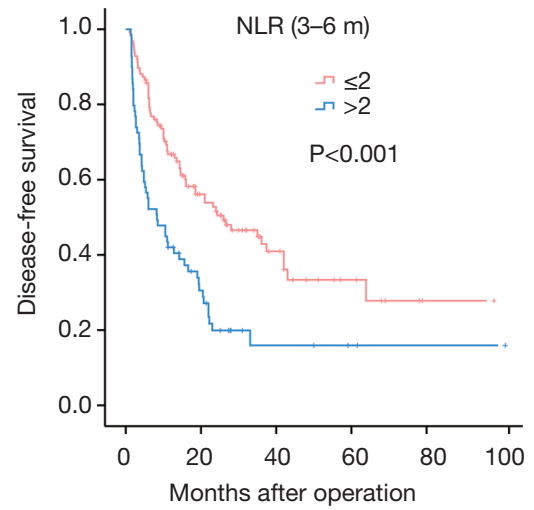

Figure 3 Kaplan-Meier curves for disease-free survival of NLRs and NLRcs in HCC patients. Pre-NLR (A), NLR (4-8 w) (B), NLR (3-6 m) (C), NLRc (4-8 w) (D) and NLRc (3-6 m) (E).

(present/absent; HR: 2.724, 95\% CI: 1.302-5.700, $\mathrm{P}=0.008$ ), BCLC stage (present/absent; HR: 2.391, 95\% CI: $1.123-$ 5.089, $\mathrm{P}=0.024), \mathrm{AFP}(>400 / \leq 400 \mathrm{ng} / \mathrm{mL}$; HR: $2.350,95 \%$ CI: $1.146-4.820, \mathrm{P}=0.020)$ and HBV-DNA $(>1,000 / \leq 1,000$ copies/mL; HR: 2.295, 95\% CI: 1.048-5.027, $\mathrm{P}=0.038)$ were found to be related to OS significantly (shown in Table 2).

In multivariate analyses, variables with $\mathrm{P}<0.05$ in univariate analyses for DFS and OS were included as adjustment factors to evaluate the prognostic values of NLRs and NLRcs. After adjustment for confounders including tumor diameter, tumor number, vascular invasion, BCLC stage, pathological differentiation and intraoperative blood transfusion, there was no significant relationship between pre-NLR and DFS (HR: 1.178, 95\% CI: $0.814-1.705, \mathrm{P}=0.385)$, while both NLR (4-8 w) and NLR (3-6 m) showed as independent prognostic factors for DFS (HR: 1.570, 95\% CI: 1.064-2.317, P=0.023; HR: $2.206,95 \%$ CI: $1.518-3.206, \mathrm{P}<0.001$, respectively).
And there was still no statistical significance between NLRc (4-8 w) and DFS $(\mathrm{P}=0.369)$ after the adjustment. As for NLRc (3-6 m) $(\mathrm{P}=0.013)$, the DFS of patients with $\geq 25 \%$ increase was significantly lower than no change (HR: 1.633, 95\% CI: $1.020-2.615 ; \mathrm{P}=0.041)$, whereas the DFS between patients with $\geq 25 \%$ decrease and no change had no statistical significance (HR: 0.784, 95\% CI: 0.512-1.200; $\mathrm{P}=0.262$ ) (shown in Table 3).

After adjustment with tumor diameter, vascular invasion, BCLC stage, AFP and HBV-DNA, there was no significant association between preNLR and OS (HR: 1.508, 95\% CI: 0.715-3.182, $\mathrm{P}=0.281)$, while both $>2 \mathrm{NLR}(4-8 \mathrm{w})$ and NLR (3-6 m) presented with lower OS than $\leq 2$ NLR (4-8 w) and NLR (3-6 m) (HR: 2.601, 95\% CI: 1.230-5.502, $\mathrm{P}=0.012$; HR: 3.939, 95\% CI: $1.807-8.583$, $\mathrm{P}=0.001$, respectively). Besides, neither NLRc (4-8 w) nor NLRc $(3-6 \mathrm{~m})$ showed statistical significance with OS $(\mathrm{P}=0.173 ; \mathrm{P}=0.073$, respectively) (shown in Table 4). 
A

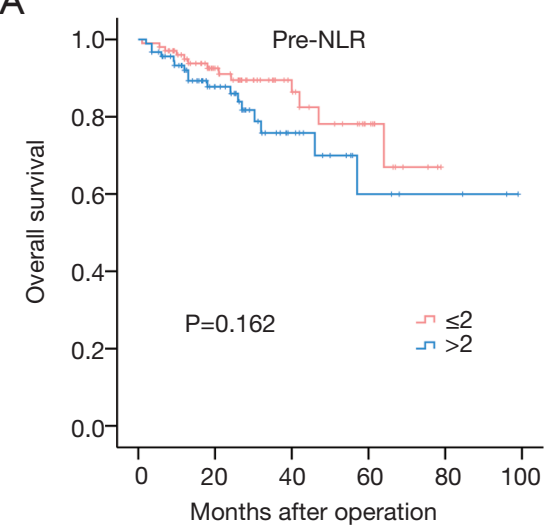

D

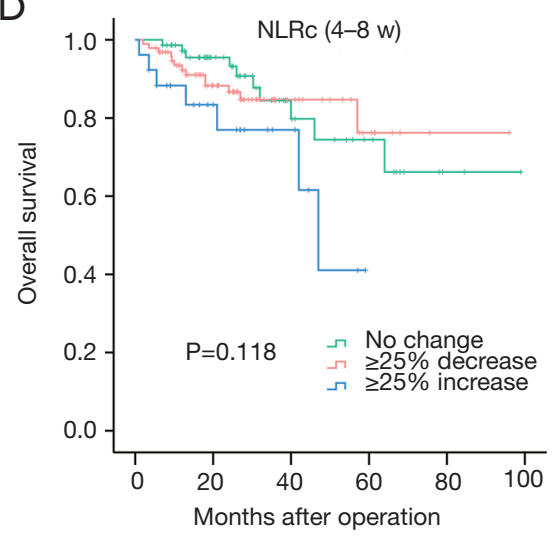

B

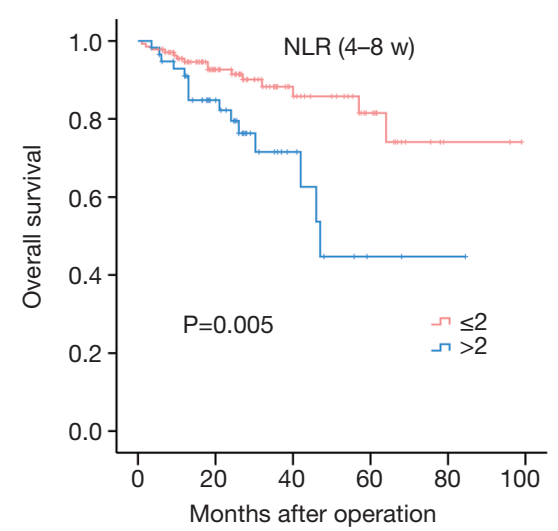

E

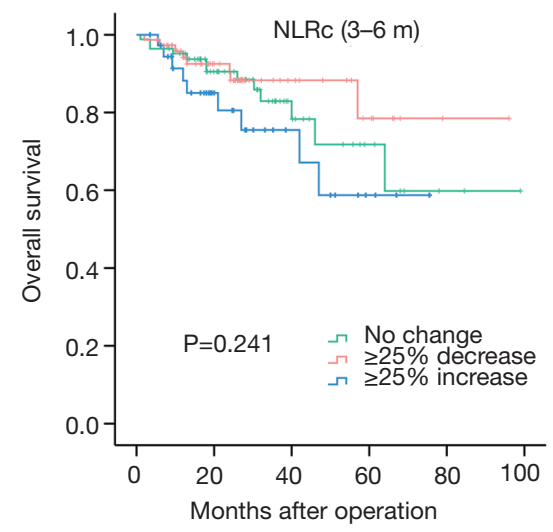

C

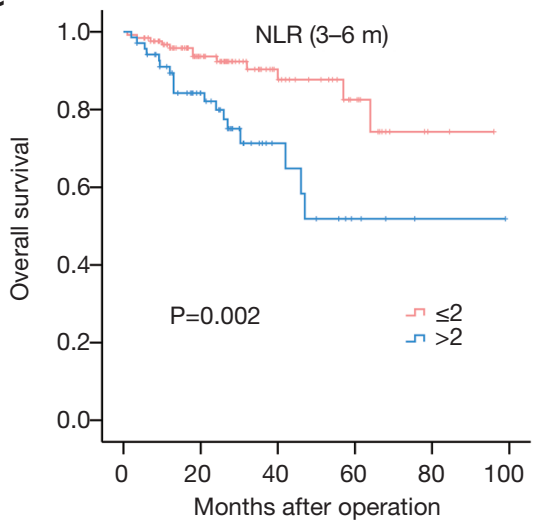

Figure 4 Kaplan-Meier curves for overall survival of NLRs and NLRcs in HCC patients. Pre-NLR (A), NLR (4-8 w) (B), NLR (3-6 m) (C), NLRc (4-8 w) (D) and NLRc (3-6 m) (E).

\section{Prognostic values of NLRs and NLRcs in HCC patients with and without cirrhosis}

Furthermore, we made a subgroup analysis for the prognostic values of pre-NLR, NLR (4-8 w), NLR (3-6 m), NLRc (4$8 \mathrm{w})$, and NLRc (3-6 m) in specific HCC patients with and without cirrhosis. And the results showed that pre-NLR was not correlated to the DFS in HCC patients regardless of the existence of cirrhosis (all $\mathrm{P}>0.05$, Figure $5 A, B$ ). The NLR (4-8 w) was also found no significant association with DFS in patients without cirrhosis $(\mathrm{P}=0.191$, Figure 5 C), while with significant association in patients with cirrhosis $(\mathrm{P}=0.010$, Figure 5D). The similar findings were also found with NLR (3-6 m), which showed no relationship with DFS in patients without cirrhosis $(\mathrm{P}=0.131$, Figure $5 E)$ while with significant relationship in patients with cirrhosis $(\mathrm{P}<0.001$, Figure $5 F)$. NLRc (4-8 w) was found with no correlation with DFS both in patients with and without cirrhosis (both $\mathrm{P}>0.05$, Figure $5 G, H$ ).
As for NLRc (3-6 m), only significant correlation was found with DFS in patients without cirrhosis $(\mathrm{P}=0.001$, Figure $5 I)$, while no correlation in patients with cirrhosis $(\mathrm{P}=0.804$, Figure 57).

In addition, pre-NLR was also not correlated to the OS in patients regardless of the existence of cirrhosis (all $\mathrm{P}>0.05$, Figure $6 A-B)$. And NLR $(4-8 \mathrm{w})$ was only found significant association with $\mathrm{OS}$ in patients without cirrhosis $(\mathrm{P}=0.032$, Figure 6C), while not in patients with cirrhosis $(\mathrm{P}=0.053$, Figure $6 \mathrm{D})$. However, NLR $(3-6 \mathrm{~m})$ was found not correlated to OS in patients without cirrhosis $(\mathrm{P}=0.349$, Figure $6 E$ ), while correlated with OS in patients with cirrhosis $(\mathrm{P}=0.001$, Figure $6 F)$. NLRc $(4-8 \mathrm{w})$ was found with correlation with $\mathrm{OS}$ in patients without cirrhosis $(\mathrm{P}=0.001$, Figure $6 G)$, while not in patients with cirrhosis $(\mathrm{P}=0.805$, Figure $6 H)$. In regard of NLRc (3-6 m), no association was found with OS in patients regardless of the presence of cirrhosis (all $\mathrm{P}>0.05$, Figure 6I, 7). 
Table 2 Univariate analysis of prognostic factors for disease-free survival and overall survival in 195 hepatocellular carcinoma patients

\begin{tabular}{|c|c|c|c|c|c|}
\hline Variables & $\mathrm{N}$ & \multicolumn{2}{|l|}{ DFS } & \multicolumn{2}{|l|}{ OS } \\
\hline Gender (female/male) & $21 / 174$ & $1.063(0.597,1.892)$ & 0.836 & $0.860(0.298,2.481)$ & 0.781 \\
\hline Age, years $(\leq 50 />50)$ & $95 / 100$ & $0.979(0.685,1.399)$ & 0.906 & $0.498(0.229,1.045)$ & 0.065 \\
\hline Cirrhosis (absent/present) & $69 / 126$ & $1.065(0.727,1.561)$ & 0.746 & $0.958(0.448,2.051)$ & 0.912 \\
\hline Tumor number (single/multiple) & $147 / 48$ & $2.051(1.397,3.012)$ & $<0.001$ & $1.110(0.475,2.595)$ & 0.810 \\
\hline Vascular invasion (absent/present) & $115 / 80$ & $1.972(1.374,2.830)$ & $<0.001$ & $2.724(1.302,5.700)$ & 0.008 \\
\hline BCLC stage $(0 \sim A / B / C)$ & $98 / 77 / 20$ & & $<0.001$ & & 0.017 \\
\hline BCLC stage (0 A/B) & & $1.863(1.259,2.757)$ & 0.002 & $1.995(0.884,4.502)$ & 0.096 \\
\hline $\begin{array}{l}\text { Intraoperative blood transfusion (absent/ } \\
\text { present) }\end{array}$ & $99 / 96$ & $1.468(1.024,2.105)$ & 0.037 & $1.186(0.576,2.439)$ & 0.643 \\
\hline AFP, ng/mL $(\leq 400 />400)$ & $130 / 65$ & $1.431(0.988,2.074)$ & 0.058 & $2.350(1.146,4.820)$ & 0.020 \\
\hline HBV-DNA, copies/mL $(\leq 1,000 />1,000)$ & $94 / 101$ & $1.309(0.914,1.874)$ & 0.142 & $2.295(1.048,5.027)$ & 0.038 \\
\hline Pre-NLR $(\leq 2 />2)$ & $103 / 92$ & $1.385(0.969,1.979)$ & 0.074 & $1.677(0.813,3.460)$ & 0.162 \\
\hline $\operatorname{NLR}(4-8 w)(\leq 2 />2)$ & $137 / 58$ & $1.742(1.193,2.544)$ & 0.004 & $2.838(1.379,5.841)$ & 0.005 \\
\hline $\operatorname{NLR}(3-6 m)(\leq 2 />2)$ & $126 / 69$ & $2.144(1.494,3.077)$ & $<0.001$ & $3.122(1.500,6.497)$ & 0.002 \\
\hline NLRc (3-6 m) (no change/ $\geq 25 \%$ increase) & & $1.640(1.037,2.594)$ & 0.034 & $1.596(0.681,3.744)$ & 0.282 \\
\hline $\begin{array}{l}\text { NLRc (3-6 m) (no change/ } \geq 25 \% \\
\text { decrease) }\end{array}$ & & $0.902(0.598,1.361)$ & 0.623 & $0.708(0.293,1.709)$ & 0.442 \\
\hline
\end{tabular}

BCLC, Barcelona clinic liver cancer; AFP, $\alpha$-fetoprotein; NLR, neutrophil-to-lymphocyte ratio; Pre-NLR, preoperative NLR; NLRc, changes of NLR.

\section{Prognostic values of NLRs and NLRcs for early and late recurrence in HCC patients}

The recurrence of HCC could be divided into two patterns with early and late phase. In the present study, the cutoff time point was set at one year after surgery, and recurrence within one year postoperatively was classified as early recurrence, while recurrence occurred after one year of surgery was named to late recurrence. Of 121 HCC patients with recurrence, 81 (66.9\%) cases belonged to early recurrence, while 40 (33.1\%) cases were late recurrence. And the results of survival analysis for early recurrence showed that pre-NLR (Figure $7 A$ ) was not correlated to early DFS $(\mathrm{P}=0.265$, Figure $7 A)$, while the NLR (4-8 w) $(\mathrm{P}=0.037$, Figure $7 B)$ and NLR $(3-6 \mathrm{~m})(\mathrm{P}<0.001$, Figure $7 C)$ were correlated to the early DFS. As for NLRcs, no correlation 


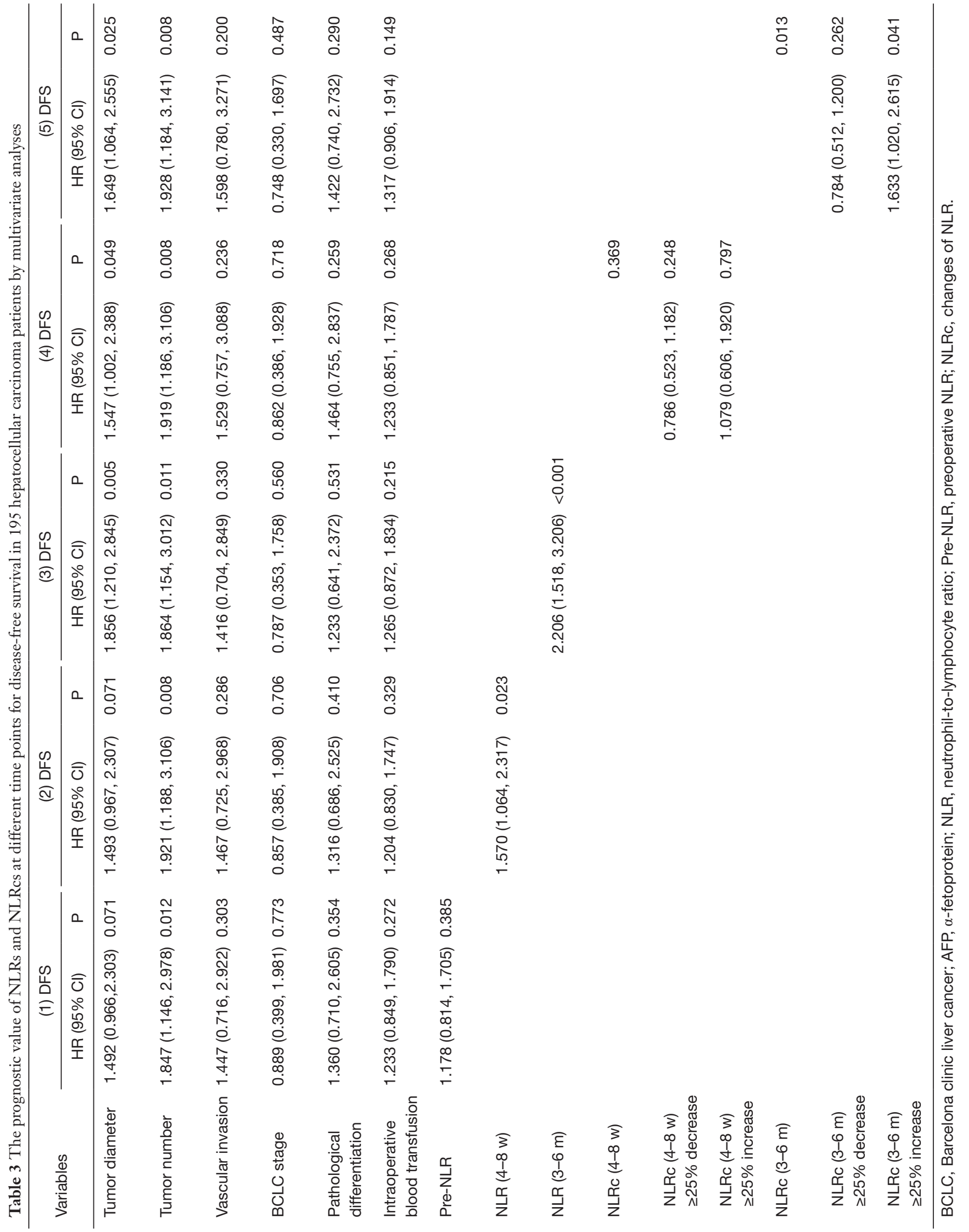



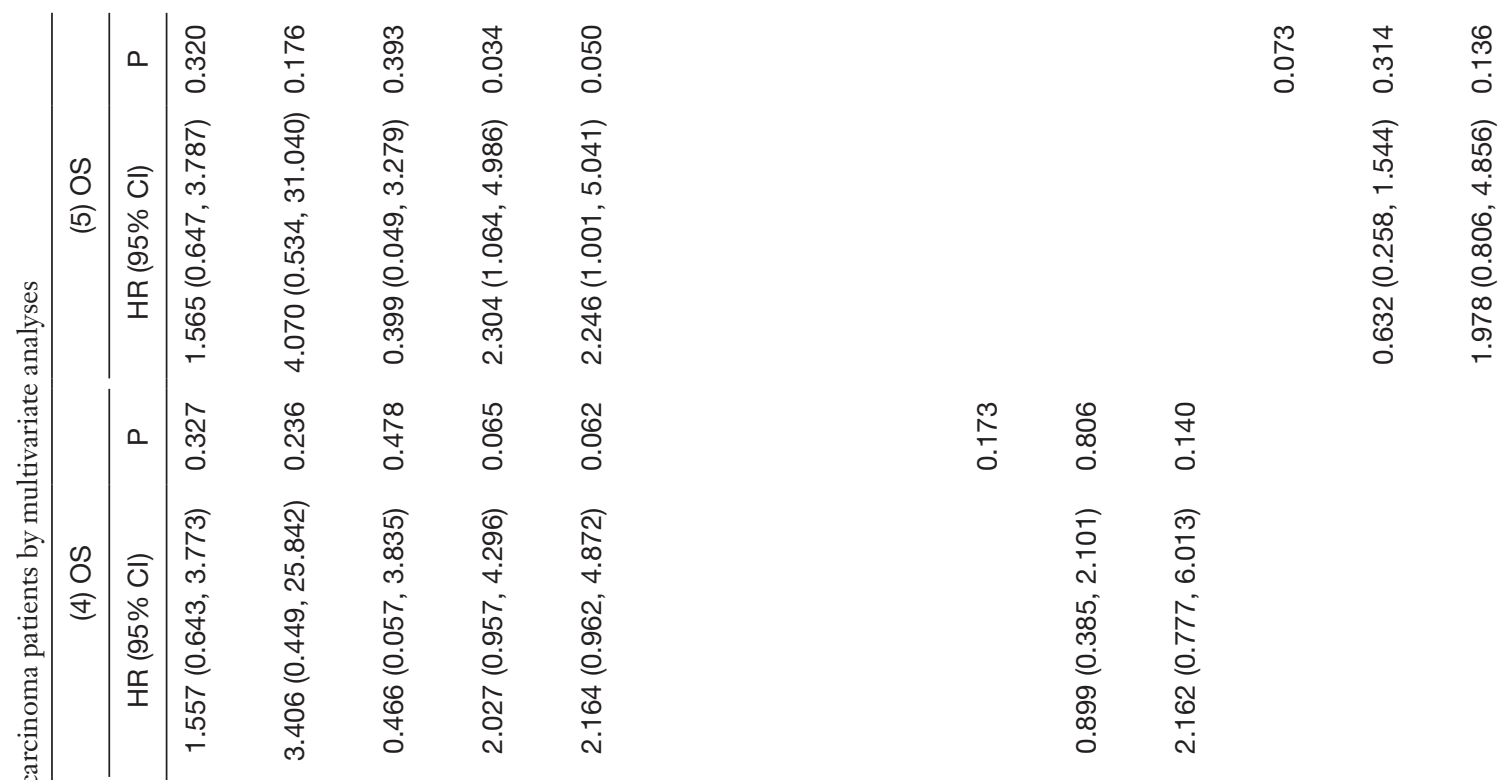

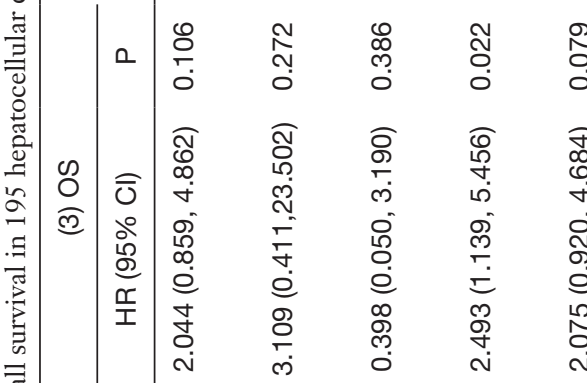

$\begin{array}{lll}\Re & 0 & 0 \\ & 0 & + \\ 0 & 0 & 0\end{array}$

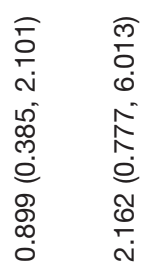

$\stackrel{5}{\circ}$

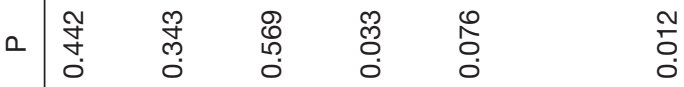

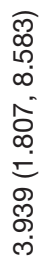

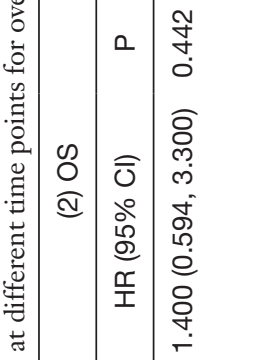

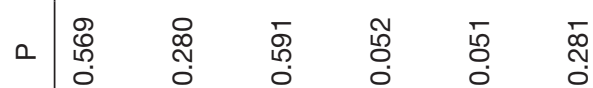

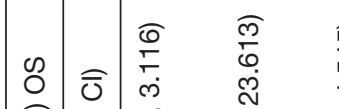

我

न त ल

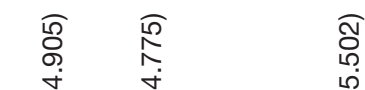

\&

¿ $\stackrel{0}{=}$

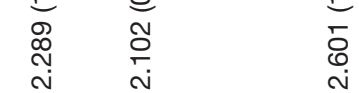

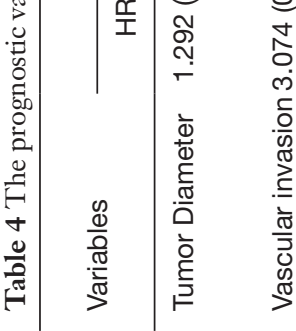

i.

華 मे के

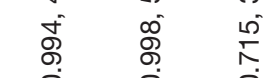

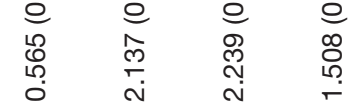

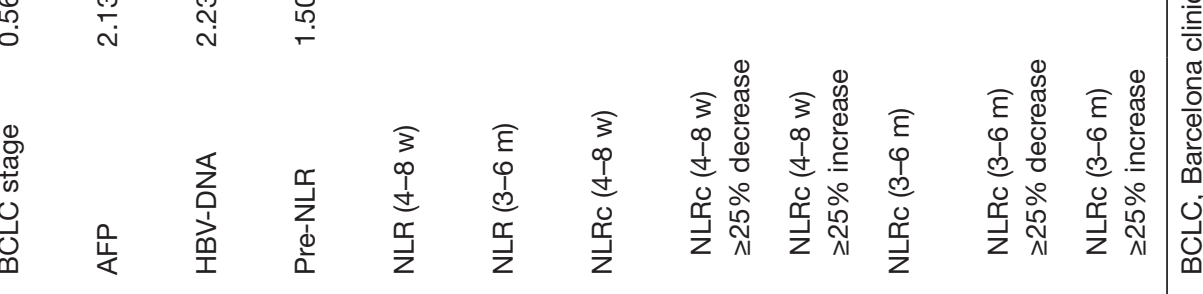



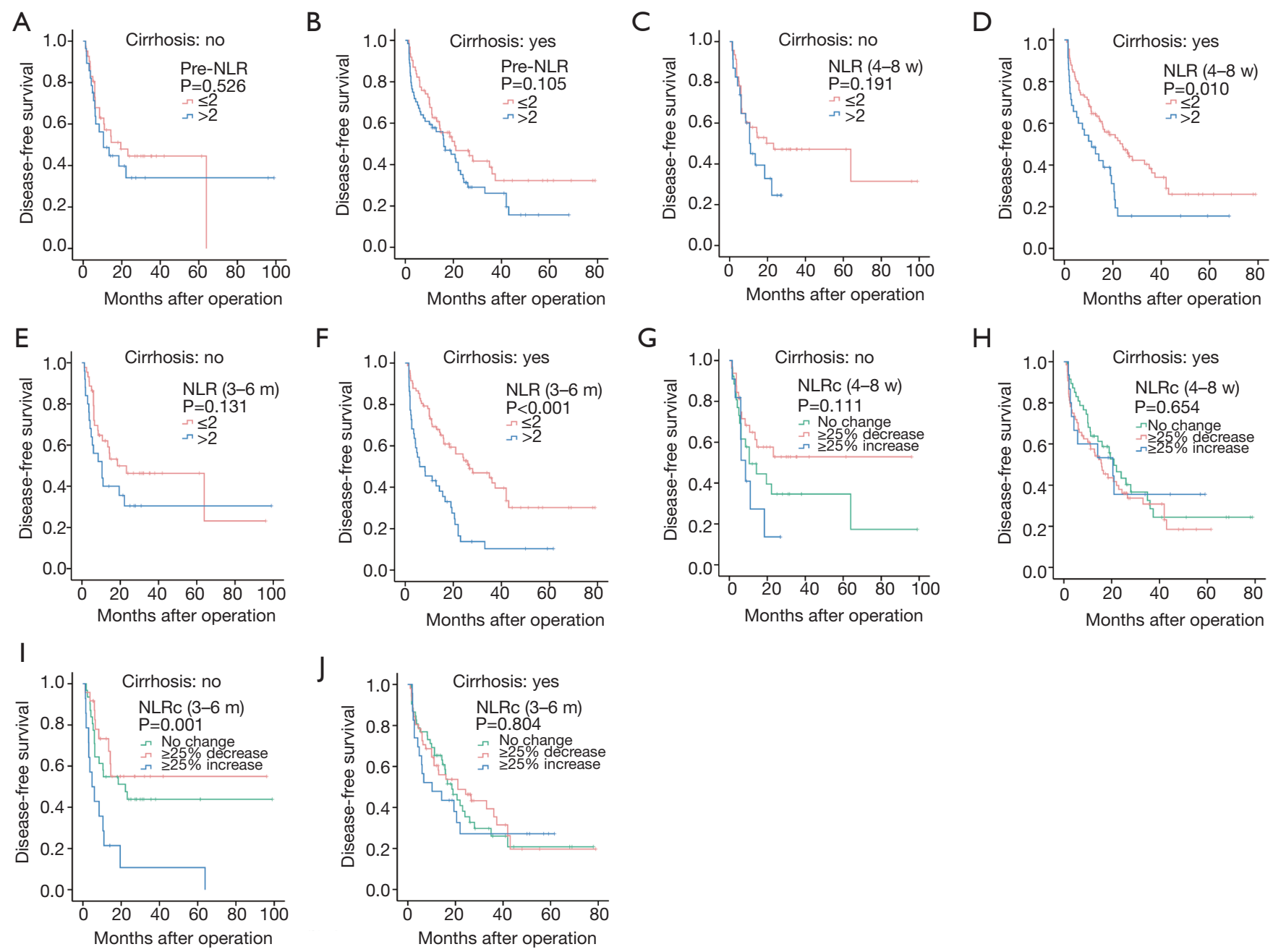

Figure 5 Kaplan-Meier curves for diseases-free survival of NLRs and NLRcs in HCC patients with and without cirrhosis. Without cirrhosis: pre-NLR (A), NLR (4-8 w) (C), NLR (3-6 m) (E), NLRc (4-8 w) (G) and NLRc (3-6 m) (I); With cirrhosis: pre-NLR (B), NLR (4-8 w) (D), NLR (3-6 m) (F), NLRc (4-8 w) (H) and NLRc (3-6 m) (J).

was found with NLRc (4-8 w) $(\mathrm{P}=0.565$, Figure $7 D)$ for early DFS, while NLRc (3-6 m) $(\mathrm{P}=0.008$, Figure $7 E)$ showed correlation. Patients with high NLR (4-8 w) and NLR (3-6 m), and with NLRc (3-6 m) $\geq 25 \%$ increase showed with worse early DFS. As for late recurrence, no significant association was found with pre-NLR $(\mathrm{P}=0.120$, Figure $8 A)$, while NLR (4-8 w) $(\mathrm{P}=0.027$, Figure $8 B)$ was found with significant association with late DFS. NLR (3-6 m) $(\mathrm{P}=0.072$, Figure $8 C$ ) was also found no significance with late DFS. As for NLRcs, neither the NLRc (4-8 w) ( $\mathrm{P}=0.990$, Figure 8D) nor the NLRc (3-6 m) $(\mathrm{P}=0.928$, Figure $8 E)$ was found significant correlation with late DFS.

\section{Discussion}

Inflammation has been demonstrated as one of the hallmarks of cancers and plays an important role in tumor development and progression $(6,24)$. And emerging evidence has shown that various inflammation-based parameters, including platelet to lymphocyte ratio (PLR), the lymphocyte to monocyte ratio (LMR), and NLR, are associated with prognosis of numerous tumors (25-30), but the underlying mechanisms have not been clarified clearly. NLR, a biomarker of systemic inflammation, has been regarded to reflect the balance between host 


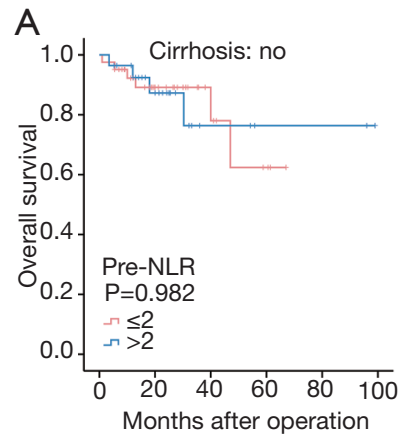

E

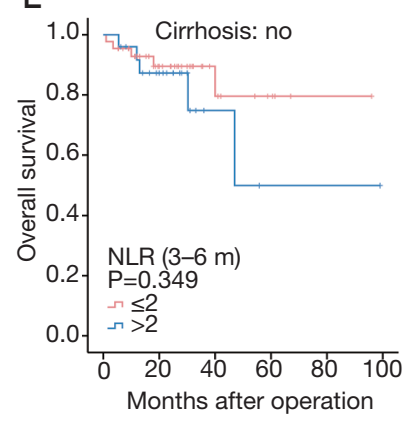

I

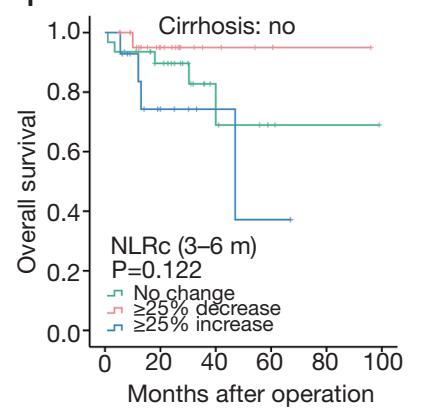

B

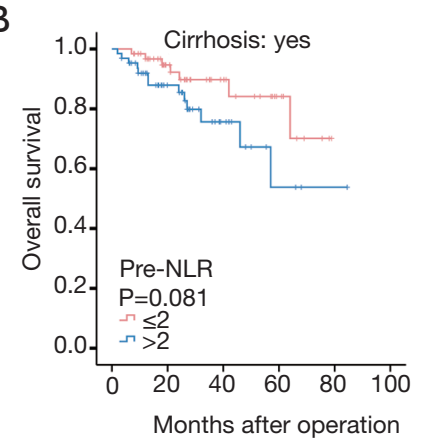

$\mathrm{F}$

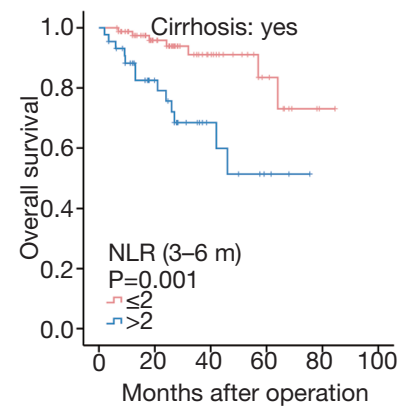

J

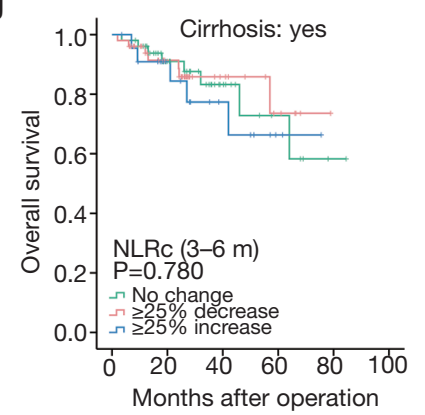

C

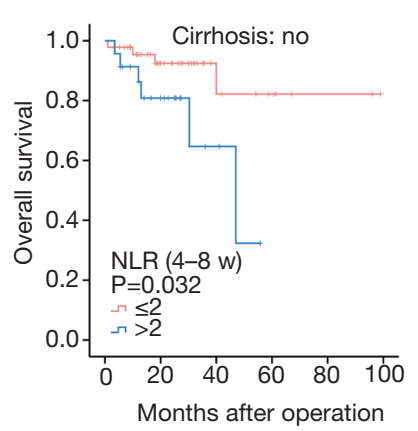

G

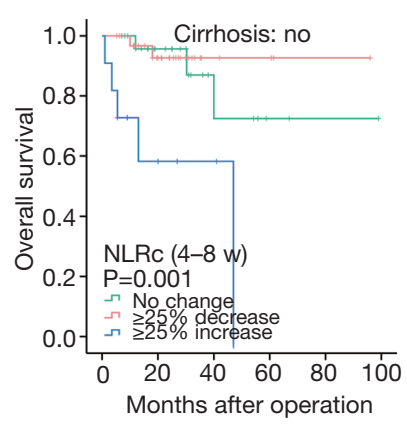

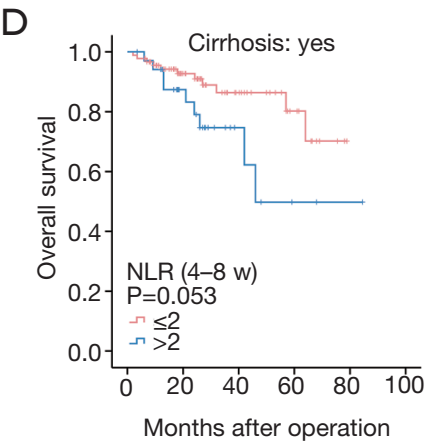

$\mathrm{H}$

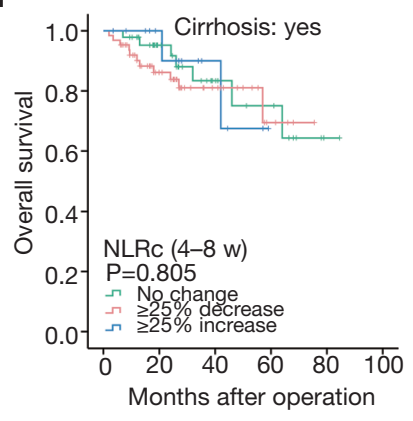

Figure 6 Kaplan-Meier curves for overall survival of NLRs and NLRcs in HCC patients with and without cirrhosis. Without cirrhosis: preNLR (A), NLR (4-8 w) (C), NLR (3-6 m) (E), NLRc (4-8 w) (G) and NLRc (3-6 m) (I); with cirrhosis: pre-NLR (B), NLR (4-8 w) (D), $\operatorname{NLR}(3-6 \mathrm{~m})(\mathrm{F}), \mathrm{NLRc}(4-8 \mathrm{w})(\mathrm{H})$ and NLRc (3-6 m) (J).

inflammatory response and immune response in the context of a malignancy. A high NLR usually shows a significant association with unfavorable survival outcomes in patients with solid cancers (31-37), as well as in HCC (7-9,21). However, most previous investigations just focused on the pretreatment values of NLR, and only limited studies have evaluated the clinical significance of post-treatment NLR or the dynamic changes of NLR, which may reflect the new accomplished status after treatment or the shift of inflammation-immune balance. In the present study, we made a comparison among pre-NLR, post-NLRs, and the changes of NLR, to evaluate the optimal prognostic predictor of DFS and OS in HCC patients receiving liver resection. To our knowledge, this is the first study compared NLRs and NLRcs at different time points. And our results suggested that the pre-NLR wasn't a significant prognostic marker of clinical outcomes for HCC patients after liver resection, whereas the higher postoperative NLRs (4-8 weeks and 3-6 months after operation) predicted worse DFS and OS in those patients. Besides, we also found that the change of NLR at 3-6 months after operation was superior to NLRc (4-8 w) on the prognostic value of recurrence. Patients with $\geq 25 \%$ increase of NLR at 3-6 months after operation had worse DFS. Subgroup analysis further demonstrated the superior prognostic significance of postoperative NLRs in HCC, especially with cirrhosis. And 
A

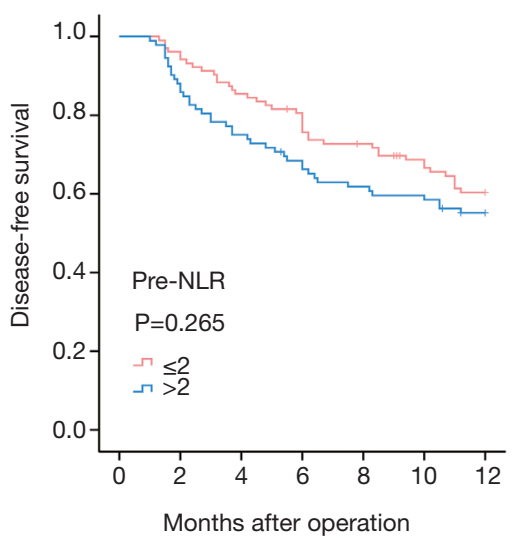

D

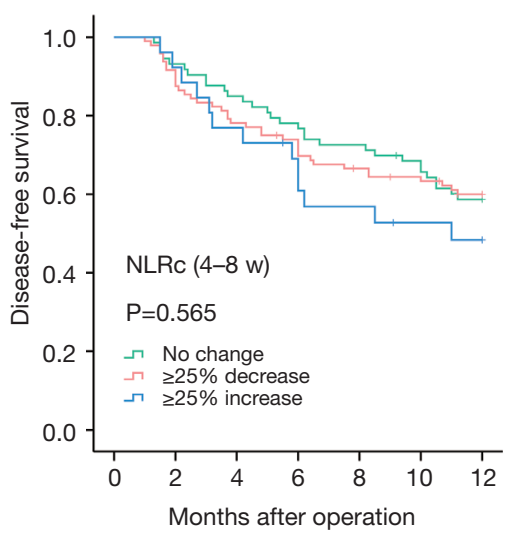

B

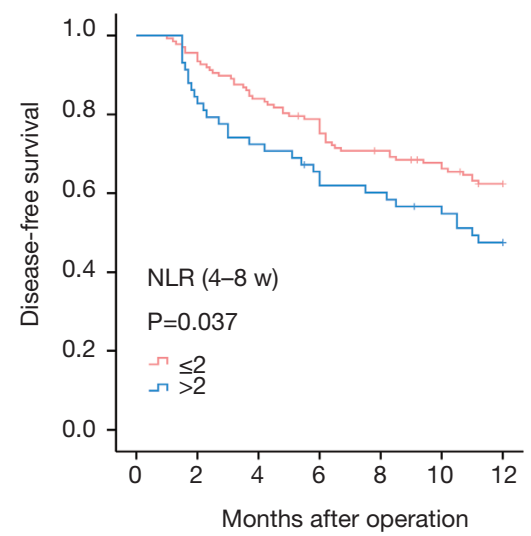

$\mathrm{E}$

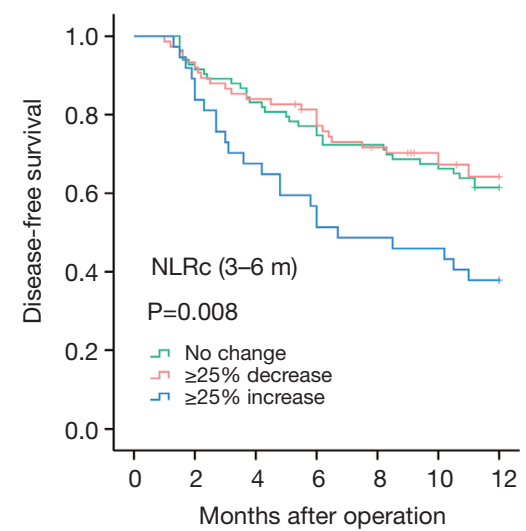

C

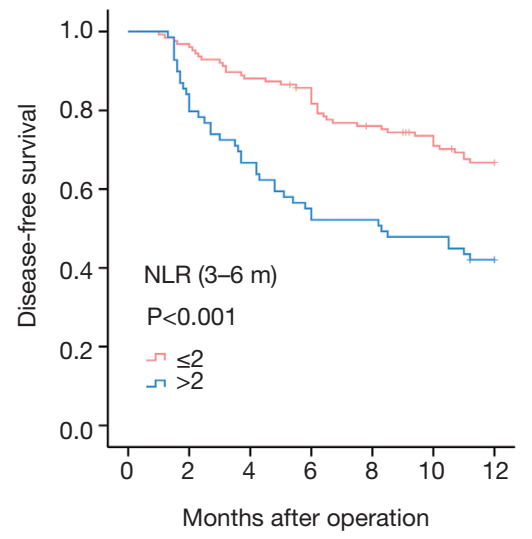

Figure 7 Kaplan-Meier curves for early diseases-free survival of NLRs and NLRcs in HCC patients. Pre-NLR (A), NLR (4-8 w) (B), NLR (3-6 m) (C), NLRc (4-8 w) (D) and NLRc (3-6 m) (E).

furthermore, postoperative NLRs also showed with better performance in predicting the early and late DFS than pre-NLR.

NLR is an easy and inexpensive parameter and could be determined by regular laboratory tests. Most previous studies have shown that pretreatment NLR is an important prognostic indicator in patients with HCC after various therapies, and elevated NLR usually predicts poor clinical outcomes in patients with different stages (7-13). Considering the potential bias from unbalanced groups with different NLR values, a method with propensity score matching (PSM) was applied by Yang et al. to adjust the clinical characteristics, and they found the preoperative NLR remained an independent predictor of recurrence for HCC patients receiving hepatectomy (30). And a recent meta-analysis also showed a positive correlation of elevated NLR with poor DFS and OS in patients with liver cancer (38). Although the molecular mechanisms associating high NLR and poor outcome remain poorly understood, the following may account for possible explanation: (I) Neutrophils play an important role in inflammation within the tumor. And neutrophilia, which could be induced by cytokines involved in cancer-associated inflammation, is able to inhibit the cytolytic activity of immune cells and exerts protumoral functions by enhancing tumor cell invasion and metastasis, angiogenesis, and extracellular matrix remodeling in cancers. (II) Evidence has proven that infiltrating lymphocytes suggest an anti-tumor immune response and associate with better response to cytotoxic treatment and prognosis in cancer patients. Thus, a lower lymphocyte count may indicate a deficient immunological defense against cancer. (III) A high NLR is also associated with a high infiltration of tumor-associated macrophages and high inflammatory cytokine production in the tumor, such as interleukin-6, interleukin-8, interleukin-17, matrix metalloproteinases, and elastases, which contribute to a 
A

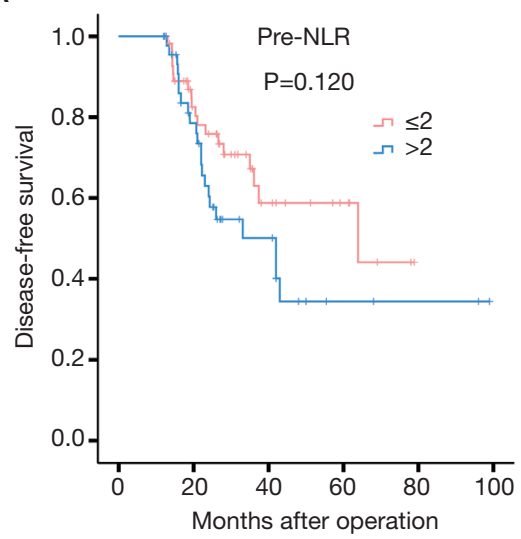

D

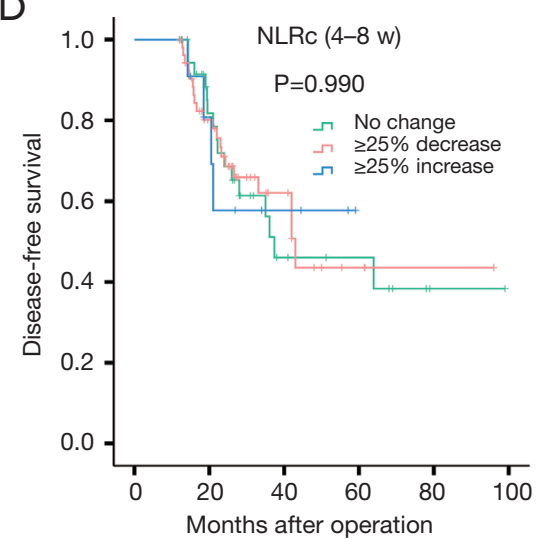

B

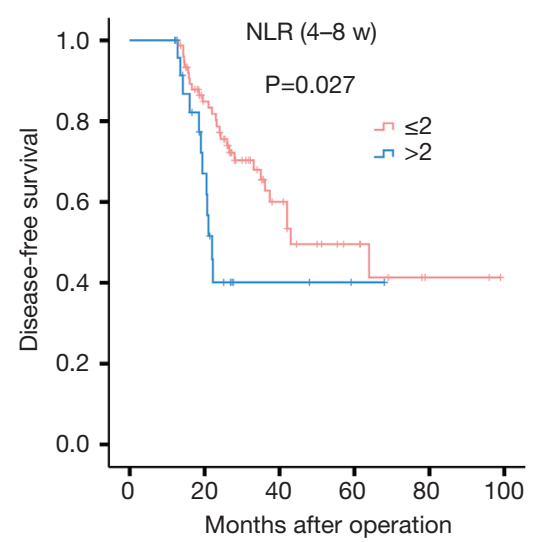

E

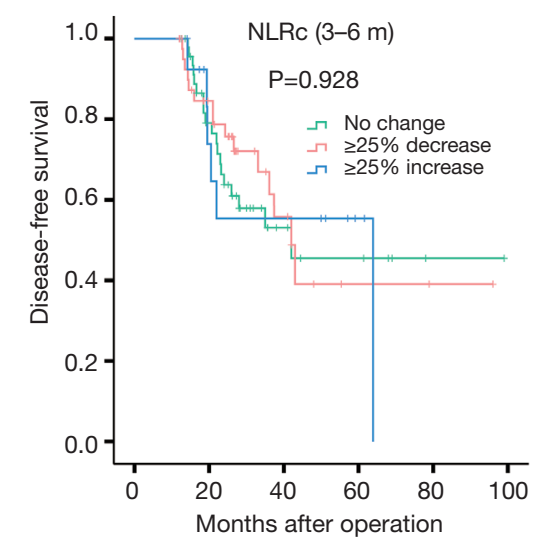

C

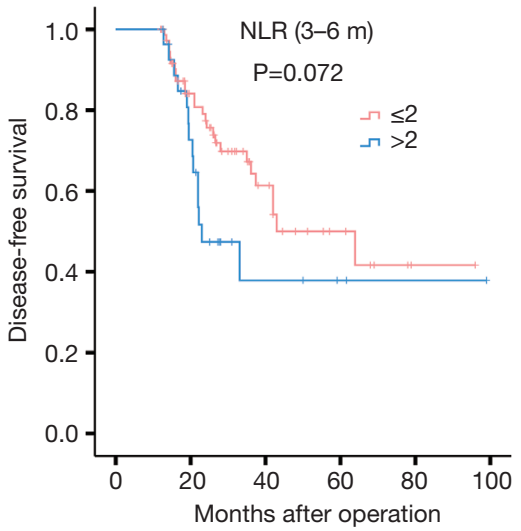

Figure 8 Kaplan-Meier curves for late diseases-free survival of NLRs and NLRcs in HCC patients. Pre-NLR (A), NLR (4-8 w) (B), NLR (3-6 m) (C), NLRc (4-8 w) (D) and NLRc (3-6 m) (E).

stimulating tumor microenvironment.

Interestingly, in the present study, we found that preNLR was not an independent prognostic factor for neither DFS nor OS in HCC patients, and high pre-NLR did not predict a worse prognosis. Except for those studies with positive results, Dan et al. and Kinoshita et al. also found that pretreatment NLR wasn't an independent predictor of recurrence-free survival (RFS)/DFS or OS in HCC patients $(14,15)$. The various cut-off values of NLR in different studies may account for some reason. A high NLR was usually determined as more than 3 or 5 in most previous studies, but the cut-off value in our study, due to the relatively low distribution of NLR in HCC patients, was set with 2 by ROC curve, which was lower than other studies.

Of note, we found that the postoperative NLR was a superior parameter for the prognosis of HCC patients. Both high NLR (4-8 w) and NLR (3-6 m) were independently correlated with poor DFS and OS in HCC patients after liver resection. Nevertheless, Chen et al. also found that the higher post-RFA NLR predicted not only worse OS, but also higher tumor recurrence rate (21). In their study, the post-RFA NLR was obtained at first follow-up visit with a median time of 3 months after RFA (range: 10-16 weeks). And recently, Hung et al. collected the preoperative NLR and NLR at recurrence after operation in HCC patients. Their results showed that high NLR at recurrence predicted worse OS and recurrence-to- death survival, and persistence of high NLR or a new elevated of NLR at recurrence showed poor outcomes (23). A meta-analysis conducted by Qi et al. showed that low post-treatment NLR was significantly associated with better overall survival of HCC patients, and decreased NLR after treatment was significantly associated with better recurrencefree or disease-free survival of HCC patients (39). The postoperative NLR might reflect the newly achieved status of systematic inflammation after the removal of tumor. 
But given the influence on the alterations of inflammation caused by surgery, the time interval of recovery to new balance remains controversial. The majority of previous studies usually use one month as the interval of recovery, but we doubt whether it is the optimal time point because of the common backgrounds with cirrhosis in HCC patients. Thus, we conducted the comparative analyses of postoperative NLR at different time points after liver resection. We found that the postoperative NLR with a comparatively long-term interval had a better predictive value. Data showed that the AUC of NLR (3-6 m) for DFS and OS was 0.656 and 0.650 , whereas the AUC of NLR (4-8 w) for DFS and OS was 0.572 and 0.621 . Hence, the postoperative NLR at 3-6 months after operation might reflect more clinical significance than NLR at one month after operation, but further clinical and experimental investigations are needed. Besides, the median of NLR (4-8 w) was lower than NLR (3-6 m), which may be related to the medications used to avoid postoperative infection.

Based on the previous investigations on preoperative and postoperative NLR with significant results, some researchers have transferred their attention to the changes of NLR after different treatments. They believed the dynamic changes may reflect the transformation of balance between host inflammatory response and immune response after therapies, but its predictive role has not been adequately explored. In patients with small HCC after curative resection, Peng et al. found that neither pre-NLR nor post-NLR was an independent factor of DFS or OS, while the NLR change showed significant predictability for the prognostic value (22). And Dan et al. also found the postoperative NLR change was a predictor for both RFS and OS, which indicated a better predictive strength of postoperative NLR change than that of preoperative NLR (15). In the present study, we also made an analysis on the prognostic significance of NLRc in HCC patients. And notably, we compared the NLRc at different time points after operation, which could show a better landscape of inflammatory characteristics at different interval after operation. In addition, patients enrolled in our study were separated into three groups according to the change ratios, and we defined a group of no change with NLR change less than $25 \%$, whereas most other studies only have two groups with an increase or decrease of NLR after treatment. Finally, we found that a $25 \%$ increase of NLR at 3-6 months after operation predicted a worse DFS than no change in HCC patients.
Nevertheless, there are several limitations in our study. Firstly, this is a retrospective analysis with data from a single center, which has some potential bias with limited cases. Secondly, the follow-up period was still limited with almost half of patients less than 24 months, which might also cause some biases. And the survival analyses need to be further evaluated in patients with long-term and valid follow-up. Thirdly, HCC patients enrolled in our study all received hepatectomy, and their BCLC stages range from 0 to $\mathrm{C}$, which may have some influence on the overall DFS and OS. But on the contrast, this population characteristic may reflect the real-world practice of treatment selection in HCC patients.

\section{Conclusions}

In conclusion, the present study demonstrates that the postNLRs and corresponding NLRcs have better prognostic significance than pre-NLR in HCC patients receiving liver resection, especially with a relatively long-term interval after operation.

\section{Acknowledgments}

Funding: This work was supported by the Guangdong Natural Science Foundation [2016A030313278, 2015A030313038, 2015A030312013]; Science and Technology Program of Guangzhou city [2014Y200200, 201604020001, 201508020262, 2014000000013, 201607010024]; Science and Technology Program of Guangdong Province [2017B020209004, 20169013]; National 13th Five-Year Science and Technology Plan Major Projects of China [2017ZX10203205-006-001]; and Guangdong Key Laboratory of Liver Disease Research [2017B030314027].

\section{Footnote}

Conflicts of Interest: The authors have completed the ICMJE uniform disclosure form (available at http://dx.doi. org/10.21037/tcr.2019.11.29). The authors have no conflicts of interest to declare.

Ethical Statement: The authors are accountable for all aspects of the work in ensuring that questions related to the accuracy or integrity of any part of the work are appropriately investigated and resolved. The study was conducted in accordance with the Declaration of Helsinki 
(as revised in 2013). The study was approved by the Ethics Committee of The Third Affiliated Hospital of Sun Yat-Sen University and written informed consent was obtained from all patients.

Open Access Statement: This is an Open Access article distributed in accordance with the Creative Commons Attribution-NonCommercial-NoDerivs 4.0 International License (CC BY-NC-ND 4.0), which permits the noncommercial replication and distribution of the article with the strict proviso that no changes or edits are made and the original work is properly cited (including links to both the formal publication through the relevant DOI and the license). See: https://creativecommons.org/licenses/by-ncnd $/ 4.0 \%$.

\section{References}

1. Forner A, Reig M, Bruix J. Hepatocellular carcinoma. Lancet 2018;391:1301-14.

2. Torre LA, Bray F, Siegel RL, et al. Global cancer statistics, 2012. CA Cancer J Clin 2015;65:87-108.

3. Llovet JM, Zucman-Rossi J, Pikarsky E, et al. Hepatocellular carcinoma. Nat Rev Dis Primers 2016;2:16018.

4. Bruix J, Reig M, Sherman M. Evidence-Based Diagnosis, Staging, and Treatment of Patients With Hepatocellular Carcinoma. Gastroenterology 2016;150:835-53.

5. Coussens LM, Werb Z. Inflammation and cancer. Nature 2002;420:860-7.

6. Grivennikov SI, Greten FR, Karin M. Immunity, inflammation, and cancer. Cell 2010;140:883-99.

7. Gomez D, Farid S, Malik HZ, et al. Preoperative neutrophil-to-lymphocyte ratio as a prognostic predictor after curative resection for hepatocellular carcinoma. World J Surg 2008;32:1757-62.

8. Halazun KJ, Hardy MA, Rana AA, et al. Negative impact of neutrophil-lymphocyte ratio on outcome after liver transplantation for hepatocellular carcinoma. Ann Surg 2009;250:141-51.

9. Huang ZL, Luo J, Chen MS, et al. Blood neutrophilto-lymphocyte ratio predicts survival in patients with unresectable hepatocellular carcinoma undergoing transarterial chemoembolization. J Vasc Interv Radiol 2011;22:702-9.

10. Mano Y, Shirabe K, Yamashita Y, et al. Preoperative neutrophil-to-lymphocyte ratio is a predictor of survival after hepatectomy for hepatocellular carcinoma: a retrospective analysis. Ann Surg 2013;258:301-5.

11. Zheng YB, Zhao W, Liu B, et al. The blood neutrophilto-lymphocyte ratio predicts survival in patients with advanced hepatocellular carcinoma receiving sorafenib. Asian Pac J Cancer Prev 2013;14:5527-31.

12. Son SH, Park EY, Park HH, et al. Pre-radiotherapy neutrophil-to-lymphocyte ratio as an independent prognostic factor in patients with locally advanced hepatocellular carcinoma treated with radiotherapy. Oncotarget 2017;8:16964-71.

13. Taussig MD, Irene KME, Mouli SK, et al. Neutrophil to lymphocyte ratio predicts disease progression following intra-arterial therapy of hepatocellular carcinoma. HPB (Oxford) 2017;19:458-64.

14. Kinoshita A, Onoda H, Imai N, et al. Comparison of the prognostic value of inflammation-based prognostic scores in patients with hepatocellular carcinoma. $\mathrm{Br} \mathrm{J}$ Cancer 2012;107:988-93.

15. Dan J, Zhang Y, Peng Z, et al. Postoperative neutrophilto-lymphocyte ratio change predicts survival of patients with small hepatocellular carcinoma undergoing radiofrequency ablation. PLoS One 2013;8:e58184.

16. Parisi I, Tsochatzis E, Wijewantha H, et al. Inflammationbased scores do not predict post-transplant recurrence of hepatocellular carcinoma in patients within Milan criteria. Liver Transpl 2014;20:1327-35.

17. Ohno Y, Nakashima J, Ohori M, et al. Followup of neutrophil-to-lymphocyte ratio and recurrence of clear cell renal cell carcinoma. J Urol 2012;187:411-7.

18. Lalani AA, Xie W, Martini DJ, et al. Change in Neutrophil-to-lymphocyte ratio (NLR) in response to immune checkpoint blockade for metastatic renal cell carcinoma. J Immunother Cancer 2018;6:5.

19. Jin F, Han A, Shi F, et al. The postoperative neutrophil-tolymphocyte ratio and changes in this ratio predict survival after the complete resection of stage I non-small cell lung cancer. Onco Targets Ther 2016;9:6529-37.

20. Min KW, Kwon MJ, Kim DH, et al. Persistent elevation of postoperative neutrophil-to-lymphocyte ratio: A better predictor of survival in gastric cancer than elevated preoperative neutrophil-to-lymphocyte ratio. Sci Rep 2017;7:13967.

21. Chen TM, Lin CC, Huang PT, et al. Neutrophil-tolymphocyte ratio associated with mortality in early hepatocellular carcinoma patients after radiofrequency ablation. J Gastroenterol Hepatol 2012;27:553-61.

22. Peng W, Li C, Wen TF, et al. Neutrophil to lymphocyte ratio changes predict small hepatocellular carcinoma 
survival. J Surg Res 2014;192:402-8.

23. Hung HC, Lee JC, Cheng CH, et al. Impact of neutrophil to lymphocyte ratio on survival for hepatocellular carcinoma after curative resection. J Hepatobiliary Pancreat Sci 2017;24:559-69.

24. Hanahan D, Weinberg RA. Hallmarks of cancer: the next generation. Cell 2011;144:646-74.

25. Huang J, Xu L, Luo Y, et al. The inflammation-based scores to predict prognosis of patients with hepatocellular carcinoma after hepatectomy. Med Oncol 2014;31:883.

26. Pinato DJ, Shiner RJ, Seckl MJ, et al. Prognostic performance of inflammation-based prognostic indices in primary operable non-small cell lung cancer. Br J Cancer 2014;110:1930-5.

27. Jiang K, Lei J, Li C, et al. Comparison of the prognostic values of selected inflammation based scores in patients with medullary thyroid carcinoma: A pilot study. J Surg Oncol 2017;116:281-7.

28. Song X, Zhu H, Pei Q, et al. Significance of inflammationbased indices in the prognosis of patients with nonmetastatic colorectal cancer. Oncotarget 2017;8:45178-89.

29. Takeuchi H, Kawanaka H, Fukuyama S, et al. Comparison of the prognostic values of preoperative inflammationbased parameters in patients with breast cancer. PLoS One 2017;12:e0177137.

30. Yang T, Zhu J, Zhao L, et al. Lymphocyte to monocyte ratio and neutrophil to lymphocyte ratio are superior inflammation-based predictors of recurrence in patients with hepatocellular carcinoma after hepatic resection. J Surg Oncol 2017;115:718-28.

31. Templeton AJ, McNamara MG, Seruga B, et al. Prognostic role of neutrophil-to-lymphocyte ratio in solid tumors: a systematic review and meta-analysis. J Natl Cancer Inst

Cite this article as: Dai T, Lin G, Deng M, Zhu S, Li H, Yao J, Li H, Liu W, Yang Y, Chen G, Wang G. The prognostic significance of neutrophil-to-lymphocyte ratio at different time points in patients with hepatocellular carcinoma receiving liver resection. Transl Cancer Res 2020;9(2):441-457. doi: 10.21037/ tcr.2019.11.29 2014;106:dju124.

32. Chua ML, Tan SH, Kusumawidjaja G, et al. Neutrophilto-lymphocyte ratio as a prognostic marker in locally advanced nasopharyngeal carcinoma: A pooled analysis of two randomised controlled trials. Eur J Cancer 2016;67:119-29.

33. Zhang L, Wang R, Chen W, et al. Prognostic significance of neutrophil to lymphocyte ratio in patients with gallbladder carcinoma. HPB (Oxford) 2016;18:600-7.

34. Bar-Ad V, Palmer J, Li L, et al. Neutrophil to lymphocyte ratio associated with prognosis of lung cancer. Clin Transl Oncol 2017;19:711-7.

35. Cho KM, Park H, Oh DY, et al. Neutrophil-tolymphocyte ratio, platelet-to-lymphocyte ratio, and their dynamic changes during chemotherapy is useful to predict a more accurate prognosis of advanced biliary tract cancer. Oncotarget 2017;8:2329-41.

36. Zhang J, Zhang S, Song Y, et al. Prognostic role of neutrophil lymphocyte ratio in patients with glioma. Oncotarget 2017;8:59217-24.

37. Lin G, Liu Y, Li S, et al. Elevated neutrophil-tolymphocyte ratio is an independent poor prognostic factor in patients with intrahepatic cholangiocarcinoma. Oncotarget 2016;7:50963-71.

38. Min GT, Li YM, Yao N, et al. The pretreatment neutrophil-lymphocyte ratio may predict prognosis of patients with liver cancer: A systematic review and metaanalysis. Clin Transplant 2018;32:e13151.

39. Qi X, Li J, Deng H, et al. Neutrophil-to-lymphocyte ratio for the prognostic assessment of hepatocellular carcinoma: A systematic review and meta-analysis of observational studies. Oncotarget 2016;7:45283-301. 